An Interactive Coal Mine Museum Visit: Prototyping the User Experience Peer-reviewed author version

HAESEN, Mieke; DE BOECK, Joan; CONINX, Karin \& RAYMAEKERS, Chris (2009) An Interactive Coal Mine Museum Visit: Prototyping the User Experience. In: 2nd International Conference on Human System Interaction (HSl'09). p. 546-553..

DOI: 10.1109/HSI.2009.5091037

Handle: http://hdl.handle.net/1942/10358 


\title{
An Interactive Coal Mine Museum Visit: Prototyping the User Experience
}

\author{
Mieke Haesen Joan De Boeck Karin Coninx Chris Raymaekers \\ Hasselt University - tUL - IBBT, \\ Expertise Centre for Digital Media, \\ Wetenschapspark 2, B-3590 Diepenbeek, Belgium \\ \{mieke.haesen,joan.deboeck,karin.coninx,chris.raymaekers\}@uhasselt.be
}

\begin{abstract}
Interactive software applications are increasingly finding their way to cultural and tourist sites. During the development of a coal mine museum on the former mine site of Beringen (Province of Limburg, Belgium), we explored the possibilities of such interactive systems, suitable for the specific site and the intended target group. In particular, we concentrated on a mobile location-based educational game allowing young visitors to gain insight in the life of the miner, and on an interactive virtual environment (VE) giving the visitor an overview of the vast site, highlighting interesting locations. In this paper we share our experiences with several prototyping techniques in the context of user-centred design (UCD). The different prototyping techniques are chosen depending on the goal and the kind of prototype. Furthermore we describe the interplay between the created prototypes and the applied prototyping techniques.
\end{abstract}

Keywords-Cultural Heritage, Model-Based User Interface Design, Prototyping, User-Centred Design, Virtual Environments.

\section{INTRODUCTION}

Mobile guides, interactive kiosks, immersive displays and virtual environments have found their way to cultural and tourist sites. These contemporary technologies are more likely to become successful systems when a user-centred design (UCD) approach is used. Careful selection of the prototypes and the prototyping techniques contributes to convincing stakeholders, to adjusting the systems for target users and to better investigating costs and benefits in feasibility studies. Though these well-known advantages of UCD and prototyping are accepted in the Human-Computer Interaction (HCI) community, it is still a challenge for the design team to define the scope of the prototypes and to pick the methodologies and techniques that are most suitable in a particular design context. In this paper, we share our experiences with prototyping activities in the context of interactive systems for a coal mine museum. The general approach, particular prototypes or techniques can inspire other researchers for their design challenges in cultural heritage applications.

The province of Limburg (Belgium) decided to open a museum on the premises of the coal mine site in the city of Beringen. The mine region in Limburg was exploited during a large part of the twentieth century, but coal mining activity ended in Beringen at the end of the eighties. The purpose of the museum is to keep alive the memories of a local industry which has been very important for the economic, social and cultural development of the region. Besides preserving the patrimonial estate, the museum aims at spreading the word about the local coal mining industry, its influence on the region and the life of miners. The province of Limburg has carried out a feasibility study with respect to the concept of the renewal and extension of the museum activities. Part of the feasibility study focused on investigating the appropriateness of particular contemporary interactive systems to enhance the accessibility of the vast museum site.

A number of considerations played a role in deciding about the prototypes to be designed for this purpose. First of all, the prototypes should provide access for the user to the most interesting assets from the large media archive about the mine. A second consideration that influenced the choice of prototypes to be developed is related to the educational mission of the mine museum. Groups of school children are regular visitors to the museum, so it is a challenge to include interactive systems which make the museum visit at the same time a pleasant and educational activity for children. Finally, the persuasive value of the prototypes is important to convince the decision makers and subsidy providers with regard to the museum extension project as such, and on the use of interactive technology to make a museum visit more attractive.

Taking into account the above three considerations, the concept for the following two interactive systems has been demonstrated through a series of prototypes. Being aware of the interest of the stakeholders in a mobile guide and the educational mission of the site, it has been decided to prototype a mobile, location-based educational game, to direct the attention of the children to objects in the museum and to present facts and figures about the life of a miner.

The concerns related to showing the vastness of the site and enhancing the accessibility of archived information can nicely be addressed in an interactive virtual environment (VE). An interactive 3D virtual model of the former mine site allows to navigate the site and interact with points of interest (e.g. a kiosk presenting multimedia information, entering a building to see pictures with details,...). After deployment of the VE, such a virtual visit to the museum can be an interesting extra facility as part of a live visit. The VE gives 
visitors a birds-eye view of the entire site, and can highlight the accessible parts and things to do during the journey. The virtual model can also fill in gaps in the real visit, for instance by giving a multimedia impression of inaccessible and dangerous parts of the mine site. In early stages, in particular during the museum preparation phase, this virtual model is also helpful to give stakeholders an impression of the site to be exploited, and how 'interactives' for the user could be spread over the renewed museum site.

The prototypes presented in this paper are not the first applications supporting historical sites and museums. A lot of resarch has been conducted on VEs [1] and mobile applications [2] [3] for cultural heritage. In this paper we focus on two assorted methodologies that have been used to realise the prototype applications, given the purpose of the prototyping activities and taking into account the considerations mentioned above. In particular, we start in the next section by describing the role of prototyping in UCD processes that adhere to the ISO standard [4]. Thereafter, in section III, we illustrate how such a process successfully hosts the prototyping activities for the creation of the mobile location-based educational game.

Next, section IV discusses the value of a UCD process namely model-based design to realise interactive high-fidelity prototypes of 3D virtual environments with a minimal coding effort. In our case study, we created the prototype of a virtual reality model of the entire coal mine site of Beringen, which is described in section $\mathrm{V}$.

Besides sharing our experiences with respect to the different prototyping techniques in the diverse prototyping stages, we want to emphasise the evolution and interplay of different prototypes. This point of view is clarified in section VI, where we illustrate the use of the virtual mine museum model to support a particular kind of prototyping of a location-based system in a lab environment.

\section{Prototyping in User-Centred Design APPROACHES}

Interactive systems, and in particular applications for cultural heritage should provide a user-friendly and attractive user interface (UI). In order to design and develop UIs that contribute to a positive user experience, user-centered design UCD is recommended. ISO 13407 [4] standardises human- and user-centred design approaches and recommends the involvement of a multi-disciplinary team that works iteratively and focuses on the end-user during the entire design process, from the early stages until the deployment of the UI.

Before specifying or designing the UI, a user and task analysis is carried out to identify the users' needs and the tasks that they perform. Based on this user and task analysis HCI engineering models are created which gradually evolve into the first prototypes. Since creativity is essential during prototyping, a designer must be part of the multidisciplinary project team involved in the prototyping stage. Designers can use models or abstract prototypes to make sure all functionalities are provided by the UI, while they use their creativity for the layout and placement of UI elements. Prototyping may occur on different levels of realism going from low to high-fidelity prototypes. Based on this level of realism, the available expertises in a project team and the characteristics of the project, a suitable tool can be used to design the prototypes [5].

A designer is responsible for the creation of low- to medium-fidelity prototypes. These prototypes are usually evaluated in a usability lab. High-fidelity prototypes should provide more functionality, which implies that besides designers, software developers are involved in the creation. The evaluation of high-fidelity prototypes can be carried out in the natural environment of the end-user during field tests and may reveal realistic usability problems. When it is not possible to involve end-users in the evaluation of prototypes, it is still recommended to evaluate prototypes within the multi-disciplinary project team or by carrying out expert evaluations.

\section{A Mobile Game For a Coal Mine Museum}

Within the scope of the VIP-lab project [6] in which we specifically aim to disseminate the benefits of a UCD approach to companies in the region, a mobile game application for educational purposes is developed. Since children seem rather uninterested while taking part of a traditional guided tour, the goal of the mobile game is to make educational excursions more interesting and interactive.

In this section we illustrate the UCD approach that was derived from the MuiCSer Process Framework [7] to realise the mobile game prototype. Fig. 1 shows the process that was adopted and the models or prototypes that resulted from each stage. The pictures between the stages present the evaluation, verification or validation of artefacts within several iterations. Artefacts created in stages (b) and (c) were verified in brainstorm sessions within the multi-disciplinary team while end-users were involved in the evaluation of the prototyping stages (d) and (e).

\section{A. Defining the Concept of the Mobile Game}

The multi-disciplinary team responsible for developing this case study consisted of computer scientists, psychologists, social scientists, field experts and a graphic designer. During a user and task analysis, eight guided tours for groups of school children (aged 8 - 14 years) were observed in four museums and zoos during a contextual inquiry [8], [9] (Fig. 1(a)). The result of this user study was a list with points of interest that influence the user experience in a positive way. It turned out that the addressed target users preferred being guided throughout the visit in a narrative way, based on a story they can identify with. Especially when users were guided by an actor playing an important character in this story, they were very interested during the tour.

Since the envisioned prototype concerned a new, locationbased system, and should be based on a story, the concept had to be clear before going on with the design. After several brainstorm sessions, the project team and the educational department of the mine museum came up with a concept for the 


\section{Mobile game for children}

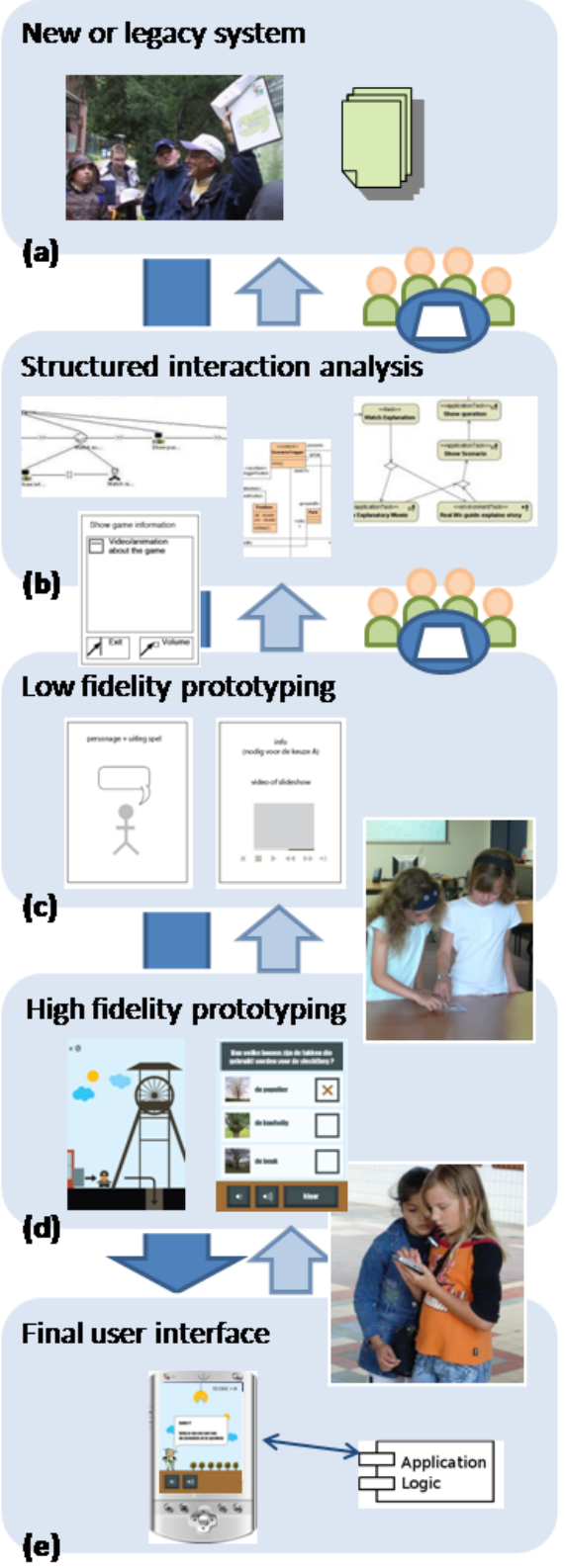

Fig. 1. The user-centred process that was adopted for the development of the mobile game.

game. A scenario ensured that all the team members had the same understanding of the game and all the points of interest, resulting from the user observations, were considered.

In the mobile application, intended for children aged 8 11 years, a virtual miner guides the children around the site. On each location in the mine museum, the miner explains to the children for what purposes this hall was used and asks them to select the equipment that he needs to collect in this hall. They have to choose the right attributes in order to prevent that the miner might be in danger. After answering the questions, animations and a video show the results. Then the children are guided to the next location.

\section{B. Structured Interaction Analysis}

During a structured interaction analysis (Fig. 1(b)), the game scenario proved to be very useful to gain a clear insight into the course of the game. The user tasks were structured in a user task model using the ConcurTaskTree (CTT) [10] notation and a few other models were created representing the connections between the application logic and the UI (e.g. application model, system interaction model). The last model that was created, was a Canonical Abstract Prototype (CAP) [11], which concerns the first presentation of the UI (Fig. 2).

\section{Low- and Medium-Fidelity Prototypes}

The scenario and some of the models (CTT and CAP) were used by the graphic designer to create the first lowfidelity prototypes. Fig. 1(c) and 2 show how the CAP gradually evolved into more detailed designs of the UI.

In order to evaluate the design in an early stage of the development, this version of the prototype was tested in a lab environment by four groups of two children (aged 8 10 years) using materials similar to what is being used in participatory approaches such as PICTIVE [12], including screens on paper and an image of a PDA (see Fig. 3(a)). Since the game concerns a location-based application, it was important to simulate the location-based effect in the lab. Two beamers that alternately showed pictures of different locations in the mine museum were used for this purpose. When the children were asked to go to the next location, they had to walk to the other projection screen.

As Fig. 3(b) shows, the test was carried out by four researchers. While two researchers were observing and recording the actions of the subjects, two other researchers simulated the role of the mobile computer in order to present the correct screens and audio. Although we expected the children to be disappointed by testing a mobile guide through dummy devices instead of popular interactive hand-held devices, it appeared that the children were very amused playing the game. The tests revealed several usability issues concerning the size of buttons, the feedback after answering a question etc. One drawback that we detected during the tests, was that the children tried to navigate on the screen to go to another location instead of walking around in the usability lab, but after a small hint, the children understood they had to physically walk to the other projection screen.
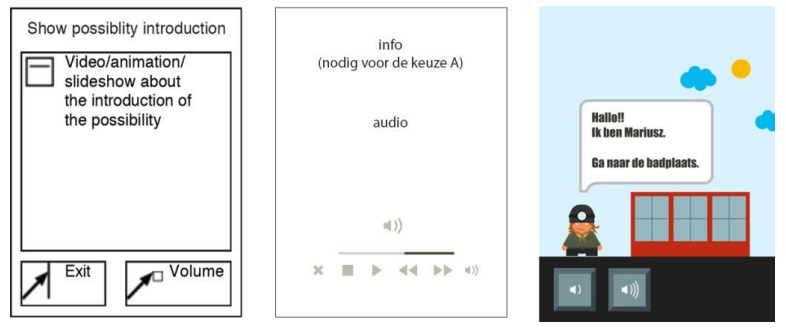

Fig. 2. A screenshot of the mobile guide for the mine museum. The CAP (left) was translated into the low-fidelity prototype (centre) which evolved into a high-fidelity prototype (right). 


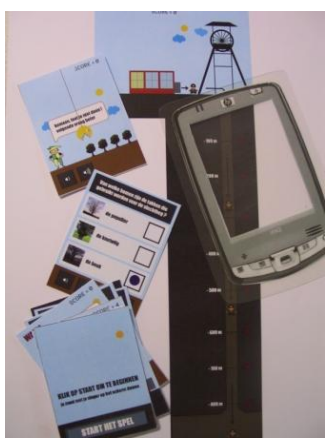

(a) The materials that were used for the evaluation of the design.

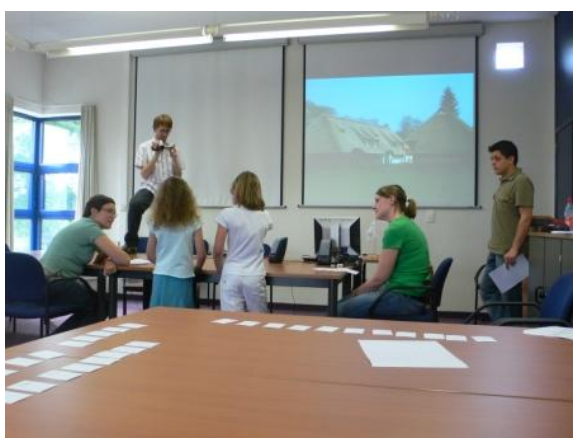

(b) Since the prototype was not deployed on a computer, several researchers were involved to facilitate the test.

Fig. 3. Evaluation of the medium-fidelity prototype.

\section{High-Fidelity Prototype}

The results of the test of the early designs were used as input for the high-fidelity prototype for the game (Fig. 1(d)). The high-fidelity prototypes were evaluated by six groups of two children (aged $8-11$ years) on the premises of the mine museum. Since the museum includes indoor locations that are currently not equipped with location-aware technology, a Wizard-of-Oz extension of the high-fidelity prototype was used. This wizard application was deployed on a second PDA that was connected to the game PDA by an ad-hoc WiFi connection. During the tests, a researcher controlled the Wizard-of-Oz application to send information about the location to the game PDA. This approach allowed to evaluate the high-fidelity prototypes in an early stage of the UCD process, independently from hardware technologies to determine the location of the game PDA. It is notable that the children did not discover that the location-awareness of the game PDA was controlled by the Wizard-of-Oz PDA.

After the lab testing of the medium-fidelity prototypes, these field tests revealed few UI problems. The problems that did occur, concerned the presentation of the content of the game and how this information was related to the location that was visited. The route between two locations sometimes was rather long, which implied that the test participants were asking questions to the observers about things they saw in the museum.

In section V-B we will show how some practical difficulties we encountered by the evaluation of the prototypes of the mobile guide may be facilitated by using a VE prototype built for the same mine museum. However, as the creation of these VEs is a long and expensive process, we propose a process that can be applied to shorten this development time. This process is shortly explained in the next section, after which in section $\mathrm{V}$, we will discuss the features of the realised VE.

\section{Prototyping a Virtual Environment using A MODEL-BASED DESIGN PROCESS}

The creation of a VE currently requires a lot of lowlevel coding effort, which implies the presence of a lowlevel code specialist within the development team. Moreover, as we are dealing with prototypes, the result is more often than not subject to frequent changes. In order to lift the creation of these VEs to a higher level of abstraction, we considered model-based user interface design (MBUID). The basic principle of MBUID is that the UI, and possibly other components of the application as well, are defined using different models. Those models have the intention to separate different concerns in different models (presentation, dialog etc.) and to abstract details by allowing reasoning on a more conceptual level. This makes the design process more accessible to non-technical team members.

Most existing MBUID design processes, however, concentrate on the design of 2D form-based interfaces [13][14][15], and lack the support required for the rich interaction that is common in VEs. For the creation of the VE prototype for the mine museum, we applied the VR-DeMo process [16], which is specifically developed to suit the needs of a MBUID process for VEs.

It falls out of the scope of this paper to describe the lowlevel details of the entire VR-DeMo process. We refer the interested reader to [17]. In the next paragraphs, we will briefly discuss the description of the rich interaction using the Notation for MultiModal Interaction Techniques, NiMMiT, as well as the available tool support, which is indispensable to apply the proposed MBUID process in practice.

\section{A. Interaction Prototyping Using NiMMiT}

One of the most important aspects in the VR-DeMo process is the way users interact with the virtual world. Usually the interaction is performed via 'interaction techniques', complex combinations of interaction styles, such as direct manipulation, gestures, speech commands, and UI elements, such as menus and dialogs. Examples of interaction techniques include object selection (e.g. ray casting) and navigation (e.g. flying vehicle).

To avoid the need to manually write code for each interaction technique, we have developed NiMMiT (Notation for MultiModal Interaction Techniques). This graphical notation allows defining the (user's) interaction by combining tasks that logically belong together. A number of predefined tasks, such as collision detection or changing the camera viewpoint, 


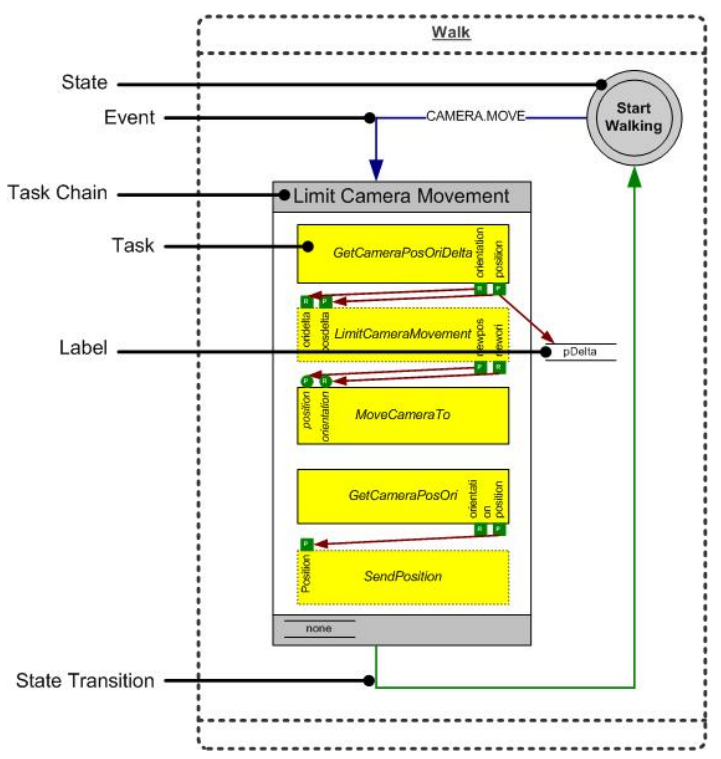

Fig. 4. Interaction diagram of a walk metaphor.

are already included, but a designer can also define custom tasks for each application. As the multimodal interaction is inherently driven by the user, the different tasks are triggered by input events, such as a button press or the movement of a tracker.

Fig. 4 shows the NiMMiT diagram for the 'walking' navigation technique, applied in the VE prototype involved. Basically, a NiMMiT diagram can be seen as a state transition diagram: at a certain time $t$, the interaction technique resides in a certain state $S$, responding to a certain set of events $E$. These events can be multimodal by nature: gesture recognition, speech recognition, button clicks,... The recognition of an event triggers an activity or task, performing 'something' in the VE application. After a succession of tasks (called a task chain) is finished, a transition to the next state is performed, letting the interaction listen to other events.

For a more thorough discussion of NiMMiT, we refer the reader to [18]. Below we want to emphasise the support that NiMMiT provides for interaction prototyping. Obviously, it is the combination of the NiMMiT notation and the provided tool support that determines the prototyping value of our model-based approach. Therefore, we continue in the next paragraphs with a brief overview of the tool support.

\section{B. Tool Support}

Model-based approaches for the creation of UIs, such as described above, are specialised processes that must be supported by a set of tools in order to be useful. The VRDeMo MBUID process for VEs is supported by CoGenIVE, which is described in more detail in [17]. In what follows, we will concisely discuss the most important features of the tool and comment on its value to prototype a VE.

CoGenIVE is built to support the VR-DeMo process, which means that it creates and manipulates the models being used in the process (as there are: the task model, dialog model, presentation model and interaction model) and finally creates an executable version of an interactive virtual VE. This provides the user with an interactive prototype in which he can navigate, select and manipulate objects according to the designer's models.

Fig. 5 shows the editor for creating the interaction description diagram using NiMMiT. The editor does not only allow the designer to define the interaction techniques, but also enforces the semantic correctness of the NiMMiT diagrams, thereby ensuring that the resulting interaction technique can be executed by the runtime system.

Undoubtedly, CoGenIVE is a valuable tool in the context of prototyping activities. In the first place, it allows for the easy creation and transformation of the specified models into a working, interactive VE. This artefact stimulates discussion with the stakeholders and within the team and invites the designers to explore alternatives and apply an iterative approach, gradually fine-tuning the prototype. Reuse of predefined NiMMiT tasks and complementing these with custom code is facilitated by the CoGenIVE editor, thus speeding up the prototyping process. Embedding the interaction description in a separate model also allows focusing on interactive aspects of the prototype and enforces a structured way of working. Finally, implementation details such as using specific devices are hidden, allowing for instance easy replacement of interaction techniques and devices. Overall, designers of a VE are stimulated to pay attention to a usable interactive system due to a decreased technical barrier.

\section{A Virtual CoAl Mine}

Applying the VR-DeMo process described in the former section, the prototype of a VE representing the mine site has been realised. Important benefits of the process and supporting tool are the shortened development time (stimulating iterations after discussions) and the considerable amount of interactivity that can relatively easy be added to the prototype.

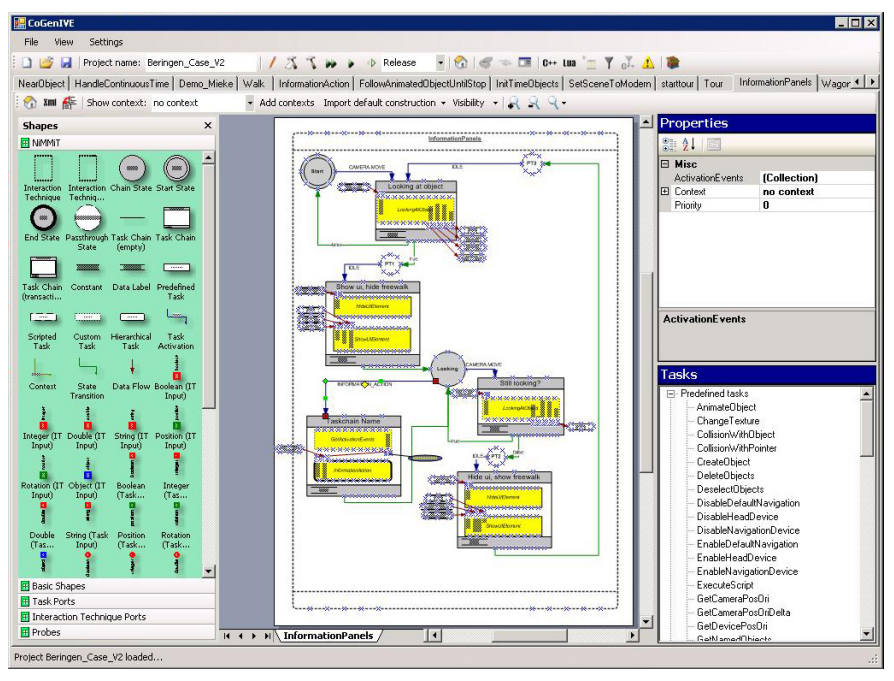

Fig. 5. Designing the Interaction Model using the NiMMiT notation. 
We have explained above that the aim of the interactive prototype is two-fold. The VE is a low-cost prototype to convince the decision makers of the possibilities of $3 \mathrm{D}$ interactive VEs in the museum. Once completed, the final version may be a valuable application for visitors to have a birds-eye view of the site and to visit inaccessible parts of the premises of the mine. We also found that the VE prototype can be seamlessly applied within the UCD process: the location-based aspect of the game is then simulated by the VE, visible on a large stereo projection screen.

In the next paragraph, we first describe the most important features of the prototype. Thereafter, we explain the coupling between the VE prototype and the high-fidelity prototype of our mobile educational game.

\section{A. Building the VE Prototype}

The VE contains a 3D model ${ }^{1}$ of the original mine site in the configuration as it was when the mine closed about 20 years ago (Fig. 6(a)). The environment is shown on a large projection wall, measuring $2.40 \mathrm{~m}$ by $1.80 \mathrm{~m}$, which allows for polarised stereo vision, as can be seen in Fig. 7. This large stereo projection offers a convincing representation of the coal mine site.

In the VE, the visitors are allowed to interactively navigate through the buildings, but they may also choose to make a system-driven virtual tour, visiting several points of interest on the site. At those locations, users can ask questions. Fig. 6(b) gives an example of the questions that can be asked when standing close to the entrance. If desired, the modelbased approach allows easy replacement of menu-selectable commands (questions) by spoken commands. When the user approaches certain locations, additional videos start to play (see Fig. 6(c)).

The prototype also contains several animations that illustrate the work flow in the mine. Fig. 6(d) depicts a model of the mine gallery where coal is picked up by a wagon and transported to the surface. Similarly an animation illustrates the chronology of the mine site (Fig. 6(e)). Users can either watch the entire time-line animation, or they can interactively navigate by operating a slider control in a dialog box. It may be expected that these interactive animations offer an additional value to the museum.

Finally, visitors may also be interested in the interior of the buildings. However, because of the current poor condition of the buildings, entering may be too dangerous. In this prototype we have chosen to apply QuickTime VR technology to show the interior. At certain places inside the building we have placed cylinders with a $360^{\circ}$ picture mapped on their interior, as can be seen from Fig. 6(f). Once entered into the QuickTime VR objects, the user can freely look around.

As stated before, the prototype application has been created using the VR-DeMo process and the CoGenIVE tool

\footnotetext{
${ }^{1}$ The 3D model representing the virtual scene is not included in the VRDeMo process, but instead can be created using any tool that exports the $\mathrm{X} 3 \mathrm{D}$ format. The final model is typically loaded by the prototype application at runtime.
}

support. Fig. 4 shows an example of a NiMMiT diagram describing the functionality for a free 'walking' through the scene. This diagram executes a number of tasks whenever the virtual camera is moved: it determines the change in the camera position, limits this change to the ground plane and applies it to the camera. Finally, some tasks are executed for synchronisation with the mobile guide, as explained in section V-B.

Most 'tasks' in a NiMMiT diagram can be chosen from a library. Only specialised tasks such as the limiting of the movements of the camera to mimic walking, have to be customly written using a scripting language or $\mathrm{C}++$ code. In the virtual reality prototype of the coal mine, the largest amount of customized code involved specific calculations (such as calculating the distance of an object) or the handling of customized data structures (for instance for the time animation).

\section{B. The Mobile Game}

Although connecting a mobile game and a VE seems not straightforward at first sight, we have been positively surprised by the additional opportunities that the availability of both prototypes offered. We decided to use the VE of the mine site, stereo-projected on a large screen, as a playground for the high-fidelity prototype of the mobile game. In this lab setting, the location-based nature is simulated by interactively navigating in the VE to trigger a particular location.

The value of the model-based approach used for the VE reveals itself in the easy extension of the VE prototype in order to communicate with the mobile device. The connection with the high-fidelity prototype of the mobile game is maintained while the user walks freely through the virtual coal mine, as defined in Fig. 4. Only two extra tasks in the NiMMiT diagram are responsible for the communication between the two prototypes. These tasks retrieve the current camera position, check if the camera is near a specific location in the virtual scene; and if applicable send this location via a wireless network connection to the mobile guide. Receiving the current location, the high-fidelity prototype of the mobile game is able to show the appropriate scene of the game to the user.

The coupling between the interactive virtual mine and the mobile educational game demonstrates an interesting interplay between both prototyping techniques. The virtual mine, being a prototype itself, is playing the role of a simulation of the real environment when evaluating the highfidelity prototype of the mobile game. As such it allows testing the location-based aspect, which turned out to be a key issue in former evaluations. In the next section we describe in more detail our findings when applying the VE technology during the user tests of the mobile guide.

\section{USING A VE TO SUPPORT UCD STAGES}

As already mentioned in the previous section, a VE can be applied in a UCD process. In this section we first describe how the integration of the high-fidelity prototype of the mobile game and the virtual coal mine contributed to the 


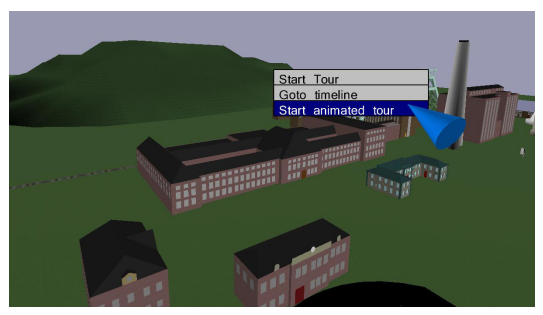

(a) Site Overview

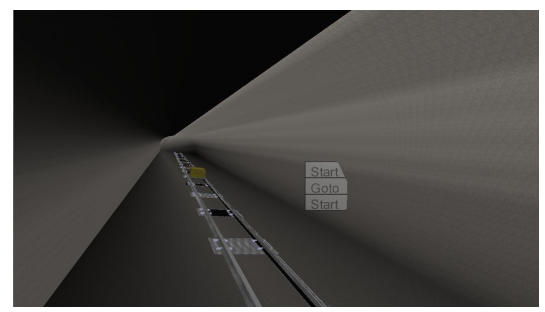

(d) The Mine Gallery

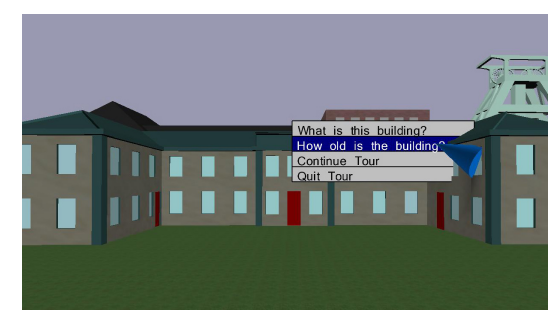

(b) Questions can be Chosen from the Menu

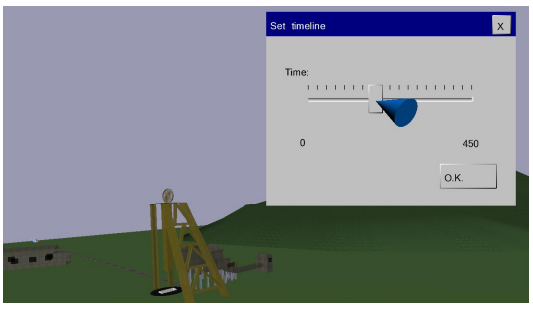

(e) Browsing Through the Time

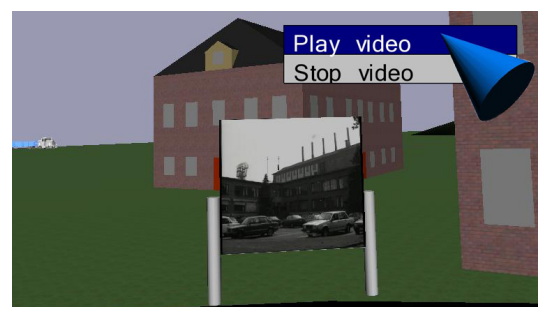

(c) Video Wall Giving Additional Information

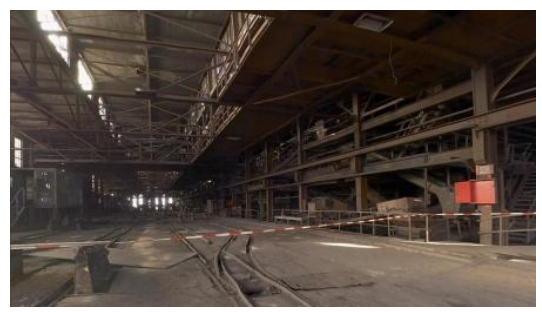

(f) QuickTime VR of the Interior

Fig. 6. Some images of the Virtual Environment

UCD process. Following, we discuss other possible benefits of using a VE in a UCD process.

\section{A. Evaluating a High-Fidelity Prototype of a Mobile Game in the Virtual Coal Mine}

The integration of the mobile game and the virtual coal mine made it possible to evaluate the high-fidelity prototype in a lab environment. We tested this evaluation method during an informal experiment. The four participating children (aged 8 - 11 years) were asked to play the mobile game, while they could virtually walk around on the mine premises (Fig. 7). As soon as the position within the VE reached a given location, the mobile guide started to play the introduction of the next part of the game. In contrast to the lab evaluation described in section III-C, the participating children immediately understood the concept of the locationbased game. They liked virtually walking around in the museum and mentioned afterwards that they would like to use a similar VE on a museum site to explore locations that are not accessible.

It is highly recommended to evaluate a prototype in the natural environment of end users. In particular when evaluating location-aware systems, the environment will contribute to the resulting user experience. Although field testing is very useful, we experienced testing high-fidelity prototypes on a museum site as very time-consuming. Moreover, observing the actions of the test users when they were walking around in the museum was very hard and several researchers needed to observe the test. Moreover, an extra researcher had to be involved to control the Wizard-of-Oz pda. Testing a first high-fidelity prototype of a mobile guide in a lab environment using a VE is less time-consuming, while locationawareness is still applied to the game and less researchers are needed during the preparation and the execution of the tests. Furthermore, the VE can be used during evaluations at earlier stages of the UCD process. By simulating realistic environments, walking speed, etc. problems are revealed early in the design.

Field testing should not be left out of a UCD approach, but this evaluation method makes it possible to evaluate prototypes of a mobile guide during the early stages of design. Furthermore, this evaluation setting is useful to carry out tests when a museum is temporarily inaccessible or under construction, and it allows to launch a user-friendly mobile guide immediately together with the opening of the museum.

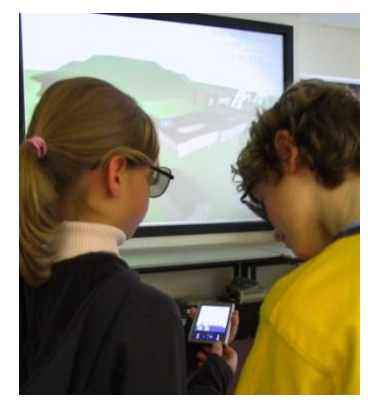

Fig. 7. Evaluation of the use of the VE in order to simulate field tests

\section{B. The Use of a VE in other UCD stages}

The successfully evaluated integration of a VE prototype to simulate field tests, opens up the way to its application within other stages of a UCD process. For instance, during brainstorm sessions in the early stages of the design, the use of the VE could help the project members to understand the museum site and to get an idea of the locations that would be part of the mobile game. Similarly, when discussing the concept of the game, ideas can be directly related to the environment using this VE. Fitting the concept of the game to the available content was one of the hardest jobs during the case study because of different expertises in the multidisciplinary team. Providing the VE to the representatives of the museum to explain the relation between the content and 
the locations of the site, will facilitate this process of fitting the game concept to the content of the museum.

Although the first tests in the usability lab (Fig. 1(d)) revealed a lot of interesting usability issues, the children did not immediately understand that they had to walk around in the usability lab. Using the VE instead of two projection screens allows them to virtually walk from one location to another. During the aforementioned informal experiment we observed that children immediately understood that they had to walk from one location to another in the virtual coal mine. Therefore a VE of a museum site is useful to add locations to a usability lab when testing location-aware prototypes.

We developed cultural heritage prototypes for the VE as well as for the mobile game, coupled these and achieved an interplay of prototyping techniques. In more general circumstances, one must consider the costs related to the possible benefits of developing the different prototypes and in particular the VE. However, rapid prototyping techniques are emerging, and we hope that our experiment provides inspiration to combine several techniques in a non-traditional way.

\section{CONCLUSION}

Interactive systems are able to make cultural heritage a rewarding experience under the condition that the systems are conceptualised in a user-centred way. In this paper we have presented the prototyping techniques we used to explore two interactive systems in this context.

The VE with a $3 \mathrm{D}$ model of the mine site of Beringen is mainly intended to give an impression of the vast site and possible 'interactives' along the tour. Interactively exploring the virtual mine model would be fascinating for visitors in the museum to get their bearings and to explore physically unaccessible parts of the site. On the other hand, the VE can be used to convince stakeholders in the project. The prototype of the interactive VE has efficiently been realised using a model-based development process incorporating the NiMMiT interaction description and supported by the CoGenIVE code generation tool.

The project developers expressed their interest for a mobile educational game to make the visit more appealing for young visitors. In order to show the benefits and possible disadvantages of this mobile application, a prototype of a game about a miner has been realised according to an iterative UCD process. We have described the evolution from paper-prototyping to high-level prototyping with attention for the location-based aspect during the evaluations.

Being able to integrate the mobile game with the VE provided us with an attractive additional technique for prototyping location-based systems. Navigating in the virtual model of the Beringen mine site turned out to be a powerful tool for the mobile game testers to get a better insight into the location-based nature of the mobile application without performing a full field test, simulating location triggers.

\section{ACKNOWLEDGMENTS}

Part of the research at EDM is funded by ERDF (European Regional Development Fund) and the Flemish Government. The VR-DeMo project (IWT 030248) is directly funded by the IWT, a Flemish subsidy organization. VIP-lab (4-BMG-II-2=37) is financed by the "Interreg Benelux-Middengebied" authorities and is co-financed by Province of Limburg (B), Province of Limburg (NL), Ministry of Economic Affairs (NL) and Ministry of Flemish Government/Economic Affairs (B).

\section{REFERENCES}

[1] N. Williams, C. Hantak, K.-L. Low, J. Thomas, K. Keller, L. Nyland, D. Luebke, and A. Lastra, "Monticello through the window," in VAST 2003: 4th International Symposium on Virtual Reality, Archaeology and Intelligent Cultural Heritage, November 2003, pp. 131-138.

[2] R. Dini, F. Paternò, and C. Santoro, "An environment to support multiuser interaction and cooperation for improving museum visits through games," in Proc. of MobileHCI 2007, 2007, pp. 169-175.

[3] J. S. Cabrera, n. F. Henar Mu A. G. Stoica, N. Avouris, Y. Dimitriadis, G. Fiotakis, and K. D. Liveri, "Mystery in the museum: collaborative learning activities using handheld devices," in Proc. of MobileHCI '05. ACM, 2005, pp. 315-318.

[4] International Standards Organization, ISO 13407. Human Centred Design Process for Interactive Systems, Geneva, Switzerland, 1999.

[5] J. Arnowitz, M. Arent, and N. Berger, Effective Prototyping for Software Makers (The Morgan Kaufmann Series in Interactive Technologies). San Francisco, CA, USA: Morgan Kaufmann Publishers Inc., 2006.

[6] K. Coninx, M. Haesen, and J. Bierhoff, "VIP-lab: A virtual lab for ICT experience prototyping," in Proc. of Measuring Behavior 2005, 2005, pp. 585-586.

[7] M. Haesen, K. Coninx, J. V. den Bergh, and K. Luyten, "MuiCSer: A Process Framework for Multi-Disciplinary User-Centered Software Engineering processes," in Proceedings of Human-Centred Software Engineering, September 2008, pp. $150-165$.

[8] H. Beyer and K. Holtzblatt, Contextual design: defining customercentered systems. Morgan Kaufmann Publishers Inc., 1998.

[9] K. Holtzblatt, J. B. Wendell, and S. Wood, Rapid Contextual Design. A How-To Guide to Key Techniques for User-Centered Design. Morgan Kaufmann Publishers, 2005.

[10] F. Paternò, Model-Based Design and Evaluation of Interactive Applications. Springer-Verlag, 2000.

[11] L. L. Constantine, "Canonical abstract prototypes for abstract visual and interaction design," in Proc. of DSV-IS 2003, ser. LNCS, vol. 2844. Springer, 2003, pp. $1-15$.

[12] M. J. Muller, "Pictive - an exploration in participatory design," New York, NY, USA, pp. 225-231, 1991.

[13] G. Calvary, J. Coutaz, D. Thevenin, Q. Limbourg, L. Bouillon, and J. Vanderdonckt, "A unifying reference framework for multi-target user interfaces," Interaction with Computers, vol. 15, no. 3, pp. 289-308, 2003.

[14] K. Luyten, T. Clerckx, K. Coninx, and J. Vanderdonckt, "Derivation of a dialog model from a task model by activity chain extraction," in Proc. of 10th Int. Conf. on Design, Specification, and Verification of Interactive Systems DSVIS 2003, Madeira, June 4-6 2003, pp. LNCS, Vol. 2844, Springer-Verlag, Berlin, 2003, pp. 203-217.

[15] G. Mori, F. Paternò, and C. Santoro, "Design and development of multidevice user interfaces through multiple logical descriptions," IEEE Transactions On Software Engineering, vol. 30, no. 8, pp. 1 - 14, August 2004

[16] J. De Boeck, J. M. Gonzalez Calleros, K. Coninx, and J. Vanderdonckt, "Open issues for the development of $3 \mathrm{~d}$ multimodal applications from an MDE perspective," in MDDAUI workshop 2006, Genova, Italy, October 22006.

[17] J. De Boeck, C. Raymaekers, and K. Coninx, "A tool supporting model based user interface design in 3d virtual environments," in Proc. of the International Conference on Computer Graphics Theory and Applications (GRAPP08), Funchal, Portugal, January 22-25 2008.

[18] J. De Boeck, D. Vanacken, C. Raymaekers, and K. Coninx, "Highlevel modeling of multimodal interaction techniques using nimmit," Journal of Virtual Reality and Broadcasting, vol. 4, no. 2, September 2007, urn:nbn: de:0009-6-11615. 